\title{
Spontaneous Radiative Efficiency and Gain Characteristics of Strained-Layer InGaAs-GaAs Quantum-Well Lasers
}

Gene Tsvid, Student Member, IEEE, Jeremy Kirch, Student Member, IEEE, Luke J. Mawst, Senior Member, IEEE, Manoj Kanskar, Member, IEEE, Jason Cai, Ronald A. Arif, Student Member, IEEE, Nelson Tansu, Member, IEEE,

Peter M. Smowton, Senior Member, IEEE, and Peter Blood, Fellow, IEEE

\begin{abstract}
The optical gain spectra, unamplified spontaneous emission spectra, and spontaneous radiative efficiency are extracted from the measurement of amplified spontaneous emission (ASE) on a single pass, segmented contact $0.98-\mu$ m-emitting aluminum-free InGaAs-InGaAsP-GaAs quantum-well (QW) laser diode. These measurements provide a baseline for which to compare higher strain InGaAs QW lasers emitting near $1.2 \mu \mathrm{m}$. The peak gain-current relationship is extracted from gain spectra and the peak gain parameter $g_{0}$ is found to agree within $25 \%$ of the value extracted using conventional cavity length analysis for $0.98-\mu \mathrm{m}$-emitting devices. The spontaneous radiative current is extracted using the fundamental connection between gain and unamplified spontaneous emission, which in turn gives an estimate of the amount of nonradiative recombination in this material system. The spontaneous radiative efficiency, the ratio of spontaneous radiative current to total current, at room temperature of 0.98- $\mu$ m-emitting InGaAs QW laser material is found to be in the range of $40 \%-54 \%$, which is $2.5-3.5$ times larger than that of highly strained InGaAs QW laser emitting near $\lambda=1.2 \mu \mathrm{m}$. Whereas the gain parameter, $g_{0}=d g / d(\ln j)$, was measured to be 1130 and $1585 \mathrm{~cm}^{-1}$ for the $0.98-\mu \mathrm{m}$ - and $1.2-\mu \mathrm{m}$-emitting materials, respectively. From the calculated below threshold current injection efficiency of $75 \%-85 \%$, we deduce that the internal radiative efficiency of the $\mathrm{QW}$ material is $\sim 20 \%$ higher than the ratio of internal radiative current to external injected current extracted directly from ASE measurements.
\end{abstract}

Index Terms-Gain spectra, InGaAs, nonradiative current, radiative current, spontaneous emission spectra, strain.

\section{INTRODUCTION}

$\mathbf{C}$ OMPRESSIVELY strained InGaAs-GaAs quantum-well (QW) active regions allow for diode lasers with high performance in the $0.9-1.2 \mu \mathrm{m}$ wavelength region. While devices in the near-IR wavelength region have demonstrated extremely

Manuscript received January 30, 2008. The work of L. J. Mawst was supported in part by the ARO MURI W911NF-05-1-0262 and in part by the National Science Foundation under Grant ECS-0355442. The work of R. A. Arif and N. Tansu was supported in part by Peter C. Rossin Assistant Professorship Funds.

G. Tsvid, J. Kirch, and L. J. Mawst are with the Electrical and Computer Engineering Department, University of Wisconsin, Madison, WI 53706 USA (e-mail: mawst@engr.wisc.edu).

M. Kanskar and J. Cai are with Alfalight Inc., Madison, WI 53704 USA

R. A. Arif and N. Tansu are with the Center for Optical Technologies, Department of Electrical and Computer Engineering, Lehigh University, Bethlehem, PA 18015 USA.

P. M. Smowton and P. Blood are with the School of Physics and Astronomy, Cardiff University, Cardiff CF24 3AA, U.K.

Digital Object Identifier 10.1109/JQE.2008.924242 high (> 70\%) total power conversion efficiencies [1], [2] questions remain as to what role nonradiative recombination plays in these structures. There are no previous reports of the overall spontaneous radiative efficiency (i.e., $\eta_{r}=J_{\mathrm{sp}} / J_{\mathrm{tot}}$ ) of high performance $0.98-\mu \mathrm{m}$-emitting InGaAs QW lasers. By increasing the compressive strain of the InGaAs-GaAs QWs, emission at wavelengths as long as $\sim 1.23 \mu \mathrm{m}$ can be achieved with threshold current densities as low as 90 $\mathrm{A} / \mathrm{cm}^{2}$ (width $=100 \mu \mathrm{m}$, and $L_{\text {cav }}=1 \mathrm{~mm}$ ) and external differential quantum efficiencies of $52 \%$ [3]. Interestingly, even with such high performance, the measured overall spontaneous radiative efficiency, $\eta_{r}=J_{\mathrm{sp}} / J_{\mathrm{tot}}$, was found to be surprisingly low $\sim 16 \%$ at room temperature (RT) [4]. A deeper understanding of the sources of radiative inefficiency of these materials is important for further improvements in the performance of these devices.

Single-pass, multisegmented amplified spontaneous emission (ASE) measurements [5] can be used to obtain the gain, absorption, and spontaneous emission spectra in real units. Integration of the calibrated spontaneous emission spectra then allows for determining the overall spontaneous radiative efficiency, $\eta_{r}=J_{\mathrm{sp}} / J_{\mathrm{tot}}$, which gives important insights into the role which nonradiative recombination plays in the active region under study. These techniques can be used as a diagnostic tool to improve the active layer material quality. It is also important to realize that the value of $\eta_{r}$ extracted from this technique is actually the product of the below-threshold (i.e., active layer carrier density is not clamped) current injection efficiency and the "true" internal $\left(\eta_{i}\right)$ or radiative efficiency of the QW material (i.e., fraction of carriers in the active layer that recombine radiatively). The below-threshold injection efficiency, $\eta_{\text {inj_below }}$ is structure, carrier density, and temperature dependent and can have values very different from the above threshold injection efficiency. Thus, if the measured $\eta_{r}$ is to give insight into the optical quality of the active region, then the dependence on $\eta_{\text {inj_below }}$ must also be considered. It is also important to note that the value of $\eta_{r}$ impacts only the threshold current density of the laser, $J_{\mathrm{th}}$, and is not a factor in the external differential quantum efficiency, $\eta_{d}$. This is because above laser threshold $\eta_{r}$ is near unity, since all carriers in the active region recombine radiatively as a result of the rapid stimulated recombination rate.

Here we report studies on the gain characteristics and spontaneous radiative efficiency of Al-free $0.98-\mu \mathrm{m}$-emitting InGaAs-InGaAsP-InGaP-GaAs, and highly strained $(\Delta a / a$ 2.6\%) 1.2- $\mu$ m-emitting InGaAs-GaAs(P)-GaAs-AlGaAs, single $\mathrm{QW}$ lasers, extracted from multisegment current injected 
TABLE I

InGaAs-InGaAsP-GaAs LASER STRUCTURE

\begin{tabular}{cccc}
\hline \hline Material & Description & Doping, $\mathbf{c m}^{-\mathbf{3}}$ & $\begin{array}{c}\text { Thickness, } \\
\mathbf{n m}\end{array}$ \\
\hline GaAs & p-Contact & $\mathrm{C}: 1 \times 10^{20}$ & 150 \\
InGaP & p-Cladding & $\mathrm{Zn}: 1 \times 10^{18}$ & 600 \\
$\begin{array}{c}\text { InGaAsP Q- } \\
\text { 1.62eV }\end{array}$ & p-Confinement & Undoped & 650 \\
InGaAs & QW & Undoped & 8.5 \\
$\begin{array}{c}\text { InGaAsP Q- } \\
1.62 \mathrm{eV}\end{array}$ & n-Confinement & Undoped & 650 \\
InGaP & n-Cladding & Si: $1 \times 10^{18}$ & 600 \\
GaAs & n-Buffer & Si: $1-2 \times 10^{18}$ & 500 \\
GaAs & n-Substrate & Si: $1-5 \times 10^{18}$ & $120 \mathrm{E} 3$ \\
\hline \hline
\end{tabular}

devices [5]. Diode lasers with a compressively strained InGaAs QW active region, emitting near $\lambda=0.98 \mu \mathrm{m}$, represent a relatively well known system and as such provide a good benchmark for comparing the gain-current characteristics obtained from gain spectra and conventional cavity length analysis [6]. To the best of our knowledge, such comparisons have not been published previously. We examine the differences in the peak gain extracted by the two methods, which is important when making predictions about the lasing properties of a media based on gain spectra. Furthermore, the gain and spontaneous emission spectra carry the information on the efficiency of pumping in producing both population inversion (gain) and spontaneous radiative recombination events.

Using the fundamental relationship between spontaneous and stimulated emission [5], [7]-[9] we extract out the spontaneous radiative current. The ratio of the spontaneous radiative current to the actual current yields the radiative efficiency, which elucidates the effect of nonradiative currents on the lasing properties of a media. Spontaneous radiative efficiency in the $40 \%-54 \%$ range is observed for InGaAs-InGaAsP-GaAs $(\lambda \sim 0.98 \mu \mathrm{m})$ devices operating near room temperature, which is significantly higher than values (13\%-19\%) observed previously for highly strained InGaAs-GaAs QWs operating at longer wavelengths $(\lambda=1.2 \mu \mathrm{m})$ [10]. Taking into account the calculated value of the below threshold current injection efficiency, we estimate that the "true" internal efficiency of the InGaAs-InGaAsP-GaAs QW material to be in the range of $47 \%-64 \%$ at room temperature.

\section{EXPERIMENTAL DETAILS}

The QW laser structures are grown by low-pressure metal-organic chemical vapor deposition (MOCVD). The layer structure for the $0.98-\mu \mathrm{m}$-emitting aluminum-free laser employs $8.5-\mathrm{nm}$ thick $\operatorname{In}_{0.2} \mathrm{Ga}{ }_{0.8} \mathrm{As} \mathrm{QW}$ with $1.62 \mathrm{-eV}$ InGaAsP barriers and waveguide, similar with that described in [11]. The full layered structure of the $0.98-\mu \mathrm{m}$-emitting laser is shown in Table I. The details of the $1.2-\mu \mathrm{m}$-emitting highly strained InGaAs QW laser have been reported in [3], with an active region consisting of a 6-nm-thick $\mathrm{In}_{0.4} \mathrm{Ga}_{0.6}$ As QW with a GaAs waveguide and $\mathrm{Al}_{0.7} \mathrm{Ga}_{0.3} \mathrm{As}$ cladding layers. The wafers were processed in multisegmented devices with the section size of $50 \times 300 \mu \mathrm{m}$,
[5]. The segments were isolated by etching narrow trenches half way into the top cladding layer and fabricating metal contacts for each segment. On the front facet an aluminum oxide film was evaporated to the thickness of $120 \mathrm{~nm}$ for antireflection purposes, estimated to have a reflectivity of less than $5 \%$. The back side of the device is intentionally scribed to spoil the cavity and minimize reflections. The devices were mounted on a TO header, epitaxy side up and gold wire bonded to the header pins. The header was clamped in a close fit to a copper block to maximize the heat conductivity. Eight section long devices were used in this study. Out of the eight sections, only the first five sections were variably pumped.

A SPEX, 1000-mm focal length, f/8, Czerny-Turner, spectrometer was used for the recording ASE spectra. A liquid nitrogen cooled germanium detector was used for power measurement. The current drive to the devices was pulsed to reduce heating with a pulsewidth of $500 \mathrm{~ns}$ and a period of $75 \mu \mathrm{s}$ using a large signal, HP8114A pulse generator. Between the pulses the device was completely off with no current flowing through it. The Ge detector time constant was in the 1-50 ms range and thus it is measuring average emission power. In addition the beam was chopped by a mechanical chopper in the range of 400 $\mathrm{Hz}$ and lock-in detection was employed. The ASE was guided by one lens, $D=50 \mathrm{~mm}, f=8 \mathrm{~cm}$, located $20 \mathrm{in}$. from the front slit of the spectrometer. Spatial filtering is done with spectrometer front slit. Data acquisition was automated by a Labview program.

For the cavity length analysis (CLA) the power was measured by a calibrated, fast InGaAs detector with integrating sphere and boxcar detection. CLA was performed on 100- $\mu \mathrm{m}$-wide monolithic laser bars of different length as well as segmented devices without spoiled cavity, wired for CLA, connecting the segments. The reason for measuring the latter is to get an estimate for the effect of inter-segment loss in the segmented devices.

\section{EXTRACTION OF GAin, SpOntaneous EMISSION AND OVERALL SPONTANEOUS RADIATIVE CURRENT SPECTRA FROM ASE}

For each current density, five ASE spectra were recorded corresponding to the length, $N * L(L=300 \mu \mathrm{m})$, of the pumped segment, $N=1,2, \ldots, 5$. The first $N$ sections were ON while 


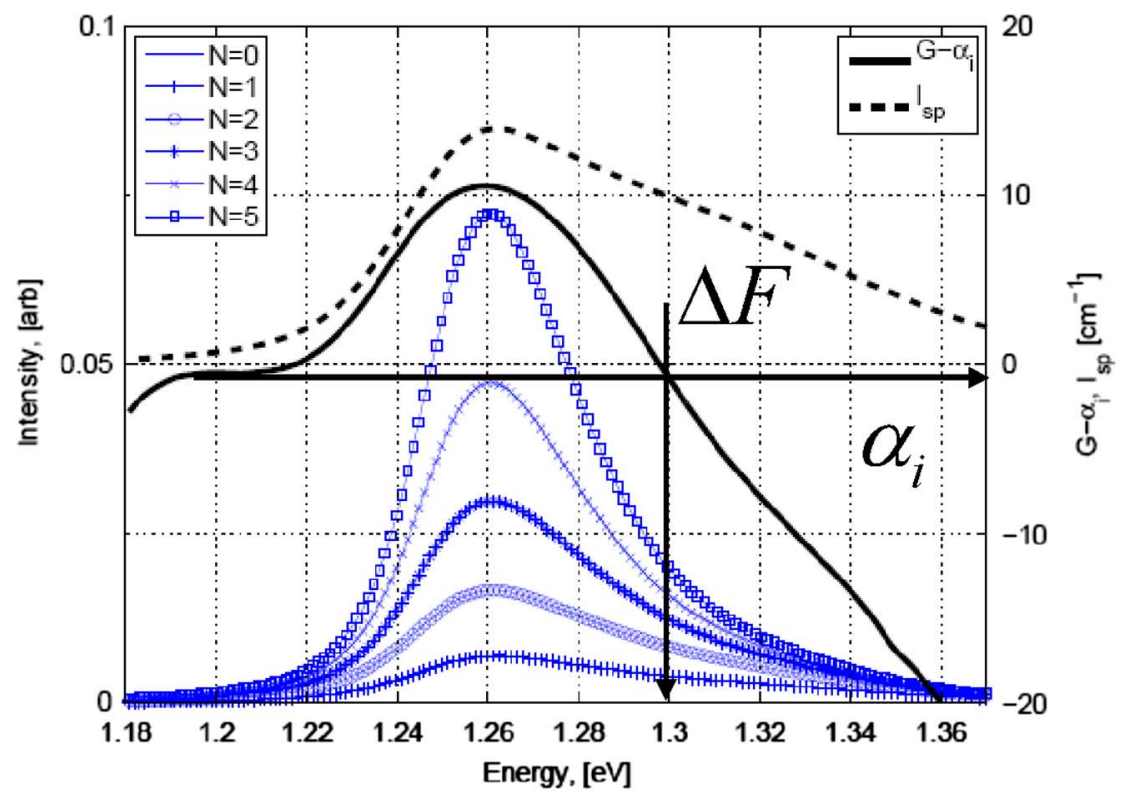

Fig. 1. Measured ASE spectra in arbitrary units for different number $N$ of turned-on segments (on the left $y$-axis). Extracted net-modal gain spectra (solid curve) and calibrated single-mode spontaneous emission (dashed curve) (on the right $y$ axis) for the $0.98-\mu \mathrm{m}$-emitting InGaAs QW laser. Method of extraction of internal loss, $\alpha_{i}$ and quasi-Fermi level separation, $\Delta F$ is shown.

the remaining $8-N$ sections were OFF. The result of a typical measurement at RT is shown in Fig. 1. The ASE spectra are fitted to the equation at each wavelength

$$
I(L)=\frac{I_{\mathrm{sp}}}{G-\alpha_{i}}\left(e^{\left(G-\alpha_{i}\right) L}-1\right)
$$

allowing extraction of the $g(h \nu)=G(h \nu)-\alpha_{i}$ and the $I_{\text {sp }}(h \nu)$ spectra. $G(h \nu)$ is the modal gain (absorption) due to electron-hole pair recombination (generation) at the energy $h \nu$ in-tune with the mode, and $I_{\mathrm{sp}}(h \nu)$ is the spontaneous emission into the mode per unit path length. All optical losses are lumped into $\alpha_{i}$. The internal loss, $\alpha_{i}$, is considered to be a function of current but not of wavelength. Spontaneous emission, $I_{\mathrm{sp}}(h \nu)$, is extracted from the fit in the units of $[\mathrm{arb} / \mathrm{cm}]$ and can be calibrated in real units of $[1 / \mathrm{cm}]$ using fundamental relationship between gain and spontaneous emission [5], [7]-[9]

$$
R_{\mathrm{sp}}(h \nu)=\frac{G_{m}(h \nu)}{\left[1-\exp \left(\frac{h \nu-\Delta F}{k T}\right)\right]} .
$$

The subscript $m$ indicates that the gain is taken in "bulk material" sense: $G_{m}(h \nu)=G(h \nu) / \Gamma$, where confinement factor $\Gamma$ is calculated from refractive indexes and layer thicknesses and given in Table II. Extraction of quasi-Fermi level separation, $\Delta F$, is shown in Fig. 1, as the energy at which $G(h \nu)=0 . T$ is taken to be room temperature and $k$ is Boltzmann's constant. Equation (2) is valid in the vicinity of the gain maximum. From the ASE fit to (1) we know $R_{\mathrm{sp}}(h \nu)$ within a constant factor. Thus, we use (2) at the gain maximum to find the scaling factor and calibrated spontaneous emission, $R_{\mathrm{sp}}(h \nu)$ in units of $\mathrm{cm}^{-1}$ into the mode. To find the overall spontaneous emission current we multiply $R_{\mathrm{sp}}(h \nu)$ by density of modes and integrate over $4 \pi$ solid angle and all frequencies, $\nu$, which gives

$$
J_{\mathrm{sp}}=\int \frac{e \cdot R_{\mathrm{sp}}(h \nu) \cdot L_{z} \cdot 8 \pi \cdot n^{2} d \nu}{\lambda^{2}}=\left[\mathrm{A} / \mathrm{cm}^{2}\right] .
$$

Here, $e$ is electron charge, $L_{z}$ is active media thickness, and $n$ is the active medium refractive index. The spontaneous radiative current obtained from (3) is plotted in Figs. 4 and 5 and will be discussed in the next section. Thus, the nonradiative current is simply the difference between total current density measured at the contact and overall spontaneous current, $J_{\mathrm{tot}}-J_{\mathrm{sp}}$. Defined as such, nonradiative current is a quantity directly accessible by the experiment although it is not only a property of material but also of the laser structure (see the discussion of injection efficiency below). The ASE measurement and extraction of all quantities is repeated for several current densities to extract gain-current relationship.

\section{RESULTS AND DISCUSSIONS}

\section{A. Gain-Current Relations}

In order to use measured gain spectra to quantify the peak gain as a function of current density, we would like to verify that it agrees well with the values extracted from conventional CLA. To this end, we compare the gain maximum versus current density curves measured by the two techniques. These gain-current curves are fitted to a phenomenological dependence, $G_{m, \max }=$ $g_{0} \ln \left(J_{\text {tot }} / J_{\text {tr }}\right)$, where $G_{m \text {, max }}$ denotes material gain spectra maximum [12], and comparison in terms of parameters $g_{0}$ and $J_{\mathrm{tr}}$ is given in Table II. We find agreement is within 25\% and is improved by comparing gain spectra directly to CLA performed on actual segmented devices. Such a comparison is useful since the devices are processed at the same time and inter-segment loss is present in both types of devices. The crude $25 \%$ agreement estimate is intended to include variability from different devices. We analyzed several devices that seem to be representative but no extensive statistical analysis was done to quantitatively characterize that variability. The internal loss $\left(\alpha_{\mathbf{i}}\right)$ extracted from gain spectra tends to be a function of drive current. 
TABLE II

MATERIAL PARAmeters FOR 0.98- AND 1.2- $\mu \mathrm{m}$ InGaAs DeTERMined By GAIN SPECTRA AND CLA ANALYSES

\begin{tabular}{|c|c|c|c|c|c|}
\hline & \multicolumn{2}{|c|}{ From Gain Spectra } & $\begin{array}{l}\text { CLA on } \\
\text { segmented } \\
\text { devices }\end{array}$ & $\begin{array}{l}\text { CLA on } \\
\text { monolithic } \\
\text { laser bars }\end{array}$ & $\begin{array}{l}\text { CLA on } \\
\text { monolithic } \\
\text { laser bars }\end{array}$ \\
\hline Material & $\begin{array}{l}0.98 \mu \mathrm{m} \\
\text { InGaAs }\end{array}$ & $\begin{array}{l}1.2 \mu \mathrm{m} \\
\mathrm{InGaAs}\end{array}$ & $\begin{array}{l}0.98 \mu \mathrm{m} \\
\text { InGaAs }\end{array}$ & $\begin{array}{l}0.98 \mu \mathrm{m} \\
\text { InGaAs }\end{array}$ & $\begin{array}{l}1.2 \mu \mathrm{m} \\
\mathrm{InGaAs}\end{array}$ \\
\hline $\begin{array}{l}\text { Device } \\
\text { Width, } \\
{[\mu \mathrm{m}]}\end{array}$ & 50 & 50 & 50 & 100 & 100 \\
\hline Reference & $\begin{array}{c}\text { Present } \\
\text { study }\end{array}$ & {$[4,10]$} & $\begin{array}{l}\text { Present } \\
\text { study }\end{array}$ & $\dagger$ & {$[3,18]$} \\
\hline$\lambda, \mu \mathrm{m}$ & 0.98 & 1.2 & 0.98 & 0.98 & 1.2 \\
\hline$\underset{\mathrm{cm}^{-1}}{\Gamma g_{0}}$ & 13.2 & 27.0 & 10.7 & 14.5 & 29.8 \\
\hline$g_{0}, \mathrm{~cm}^{-1}$ & 1119 & 1588 & 911 & 1229 & 1750 \\
\hline $\begin{array}{c}J_{t r}, \\
\mathrm{~A} / \mathrm{cm}^{2}\end{array}$ & 113 & 91 & 83 & 73 & 59 \\
\hline$\Gamma$ & 0.0118 & 0.017 & 0.0118 & 0.0118 & 0.017 \\
\hline $\begin{array}{l}\text { th, } \\
\mathrm{A} / \mathrm{cm}^{2}, \\
1 \mathrm{~mm} \text { long }\end{array}$ & & & 260 & 169 & 130 \\
\hline$\alpha_{i}, \mathrm{~cm}^{-1}$ & $0-2$ & $0-10$ & 0.82 & 0.74 & 6 \\
\hline $\begin{array}{l}\text { Radiative } \\
\text { Efficiency } \\
\text { Injection } \\
\text { efficiency } \\
\text { (extracted } \\
\text { above } \mathrm{I}_{\text {th }} \text { ), } \\
\eta_{i}, \%\end{array}$ & 47 & 16 & 67 & 83 & 79 \\
\hline
\end{tabular}

$\uparrow \mathrm{CLA}$ data on $0.98 \mu \mathrm{m}$ InGaAs is courtesy of Alfalight Inc, private communication.

For typical current density of $267 \mathrm{~A} / \mathrm{cm}^{2}$, the internal loss $\left(\alpha_{\mathrm{i}}\right)$ is in the range of $0-2 \mathrm{~cm}^{-1}$ while $1 \mathrm{~cm}^{-1}$ is a typical value determined from CLA for the $\alpha_{\mathrm{i}}$. Unfortunately it is difficult to measure the internal loss from the gain spectra more accurately to allow for a more quantitative comparison. Specifically, it is difficult to achieve with $g \sim 1 \mathrm{~cm}^{-1}$ and the segment length of $L=300 \mu \mathrm{m}$, since the relative output intensity accuracy $\Delta I / I$ should be on the order of $(g L)^{2} \sim 0.1 \%$ [cf. (1) for $g L \ll 1$ ], which appears to be below resolution limit and dynamic range of our power measurement system.

Based on the gain and spontaneous emission spectra measured with segmented devices, we can compare the properties of the $\mathrm{In}_{x} \mathrm{Ga}_{1-x}$ As ( $x=0.2$ and 0.4$)$ QW laser material. We see that the prominent differences of the highly strained QW material are a larger gain parameter, $g_{0}$, and lower transparency current density, $J_{\mathrm{tr}}$, as shown in Fig. 2 and Table II. We attribute the lower transparency current density to the beneficial effect of strain on the energy band structure, through equalization of conduction and valence band density of states [13], [14]. The compressive strain reduces the density of states, which is expected to lead to a reduction in the $g_{0}$ value. However, other factors such as differences in QW width and emission wavelength also influence the value of $g_{0}$, and may account for the increase observed in the $1.2-\mu \mathrm{m}$-emitting InGaAs QW $\left(d_{\mathrm{QW}}=6\right.$

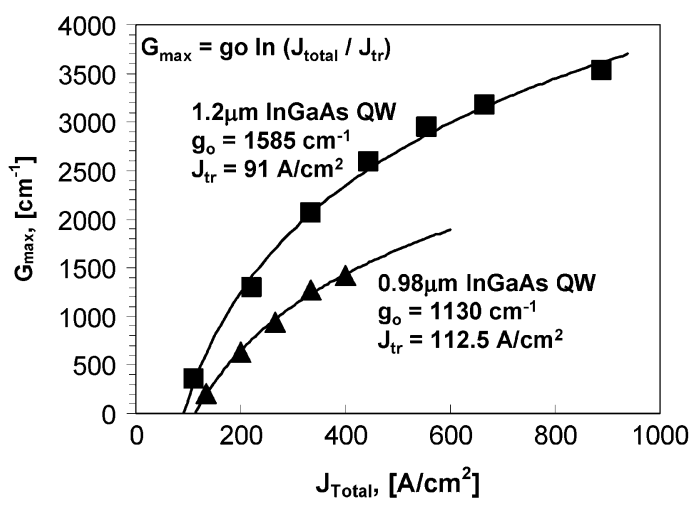

Fig. 2. Material gain maximum versus total external injected current density determined from gain spectra measurement for $0.98-\mu \mathrm{m}$ and $1.2-\mu \mathrm{m} \mathrm{InGaAs}$ QWs.

$\mathrm{nm}$ ) compared with that of the $0.98-\mu$ m-emitting InGaAs QW $\left(d_{\mathrm{QW}}=8.5 \mathrm{~nm}\right)$.

The higher $g_{0}$ parameter of the 1.2- $\mu$ m-emitting $\mathrm{In}_{0.4} \mathrm{Ga}_{0.6} \mathrm{As} \mathrm{QW}$ also correlates with the narrower width of the gain spectrum. In Fig. 3, we plot the gain full-width at half-maximum (FWHM) relative to gain maximum energy versus gain value. We see that the highly strained InGaAs 


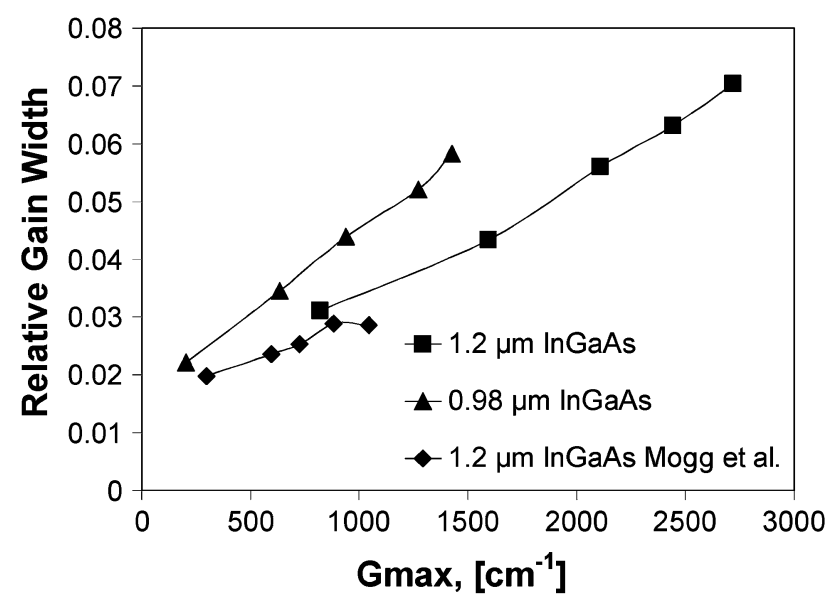

Fig. 3. Relative gain width at half maximum (relative to the energy of gain maximum) versus material gain maximum determined from gain spectra measurement for $0.98-\mu \mathrm{m}$ and $1.2-\mu \mathrm{m} \mathrm{InGaAs}$ QWs. For comparison data from [21] is shown (diamond markers).

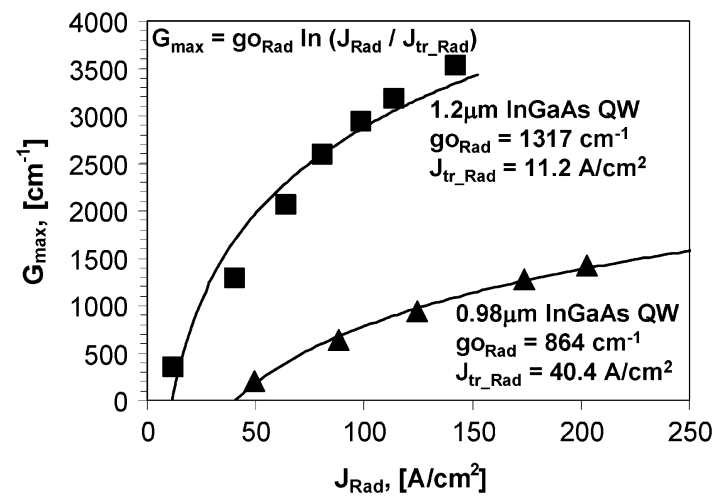

Fig. 4. Material gain maximum versus spontaneous radiative current density determined from gain spectra measurement for $0.98-\mu \mathrm{m}$ and $1.2-\mu \mathrm{m} \mathrm{InGaAs}$ QWs.

QW achieves a given gain value with relatively narrower gain spectrum, allowing for higher gain to be achieved for typical pumping levels. We expect a material with a narrower gain to have a higher $g_{0}$ since from (2) and (3) it follows that such a material achieves a given value of gain with a lower radiative current. Thus, for a fixed nonradiative current, a material with a narrower gain spectrum will achieve a given gain value with a lower total current, or equivalently, it has a higher $g_{0}$. Similar to what is observed from Fig. 2, the higher strain InGaAs QW achieves a given gain with a lower radiative current, $J_{\mathrm{rad}}$, as can be seen from Fig. 4. This is one factor leading to the lower threshold current densities of the highly strained $\lambda=1.2 \mu \mathrm{m}$ InGaAs QW lasers for the same device geometry. Note that the radiative transparency current density for the highly strained InGaAs QW is exteremely low, $J_{\text {tr_rad }}=11.2 \mathrm{~A} / \mathrm{cm}^{2}$, indicating that significant improvement in device performance is possible if nonradiative recombination can be further reduced.

The radiative current of the $\lambda=0.98 \mu \mathrm{m}$ InGaAs QW constitutes a higher fraction of the total current than for the case of the highly strained $\lambda=1.2 \mu \mathrm{m} \mathrm{InGaAs} \mathrm{QW}$, that is, it has a higher

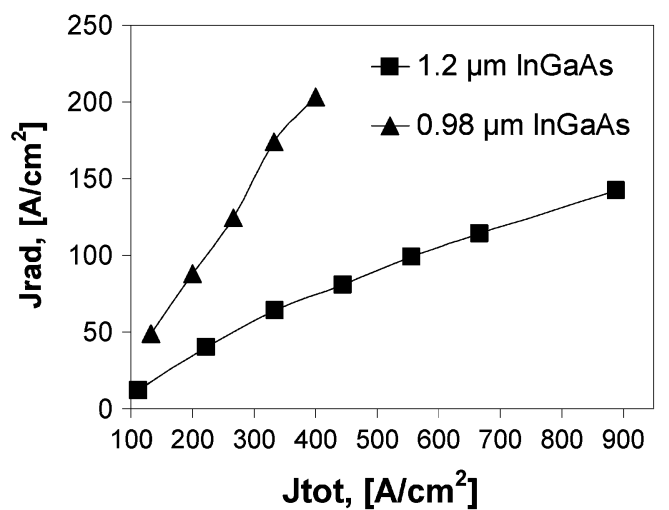

Fig. 5. Internal spontaneous radiative current density versus total external injected current density at the contact determined from gain spectra measurement for $0.98-\mu \mathrm{m}$ and $1.2-\mu \mathrm{m} \mathrm{InGaAs}$ QWs.

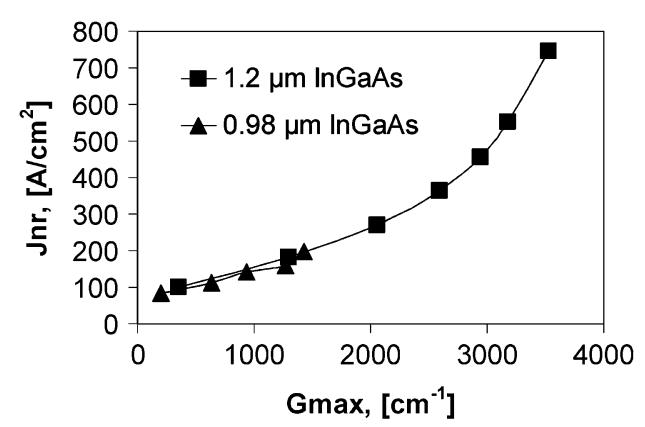

Fig. 6. Nonradiative current density versus material gain maximum for $0.98-\mu$ $\mathrm{m}$ and $1.2-\mu \mathrm{m} \mathrm{InGaAs} \mathrm{QWs.}$

spontaneous radiative efficiency, as shown in Fig. 5. Therefore, the $0.98-\mu \mathrm{m}$-emitting InGaAs QW performs closer to the fundamental limit determined by its energy band structure, which would be achieved if the nonradiative current is eliminated. Although the radiative efficiency of the 1.2- $\mu$ m-emitting InGaAs QW is lower, its QW active region actually reaches a given gain with about the same amount of nonradiative current as the $0.98-\mu$ m-emitting InGaAs QW, due to the more favorable band structure and lower total current density, $J_{\text {tot }}$, as shown in Fig. 6. Thus, the improved gain from the higher strain $\lambda=1.2 \mu \mathrm{m} \mathrm{In-}$ GaAs QW, together with the lower transparency current density, results in a lower threshold current density laser for the same device geometry.

\section{B. Internal Radiative Efficiency}

In order to properly interpret the extracted value of the radiative efficiency, we need to consider the influence of the current injection efficiency. The current injection efficiency $\left(\eta_{\text {inj }}\right)$ of QW lasers is defined as the fraction of the injected current that recombines, both radiatively and nonradiatively, in the $\mathrm{QW}$-active region of the laser. As pointed out by Smowton and Blood [15] for the case of visible-wavelength InGaP QW lasers, the at-threshold (including below-threshold) and above-threshold current injection efficiency can have very distinct values. In general, the at-threshold and above-threshold current injection efficiency of QW lasers are conceptually very different [6], [16]. A 


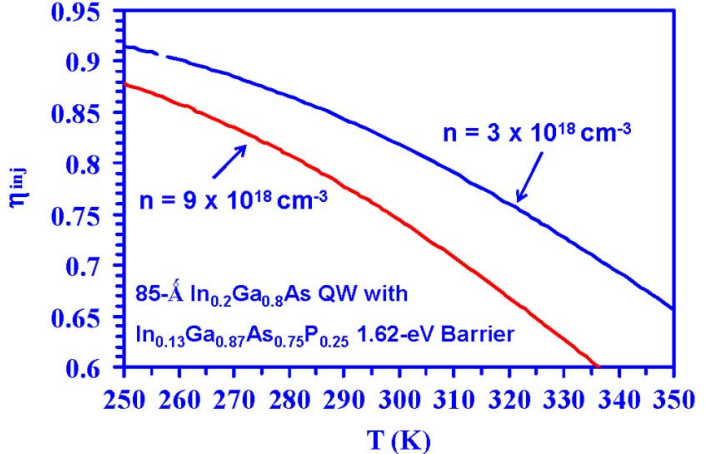

Fig. 7. Calculated below threshold injection efficiency for Al-free $0.98-\mu \mathrm{m} \mathrm{In}$ GaAs QW.

general equation for current injection efficiency for the belowand at-threshold conditions was given in [17]:

$$
\eta_{\text {inj_below }} \cong \frac{1}{\left[1+\frac{\tau_{\text {bw }}}{\tau_{b}} \cdot\left(1+\frac{\tau_{\mathrm{QW} \text { total }}}{\tau_{e}}\right)\right]}
$$

where $\tau_{\mathrm{bw}}$ is the total carrier capture time from the $\mathrm{SCH} / \mathrm{bar}$ rier to $\mathrm{QW}, \tau_{\mathrm{QW} \text {-total }}$ is the recombination lifetime in the $\mathrm{QW}$, $\tau_{e}$ is the carrier thermionic emission escape time from the $\mathrm{QW}$ to the barrier region, $\tau_{b}$ is the total recombination lifetime of the carriers in the barrier/SCH region. The simulated $\eta_{\text {inj_below }}$ values for the InGaAs-InGaAsP QW laser structure are shown in Fig. 7 as a function of temperature and carrier density. Note that at $300 \mathrm{~K}$, even if the radiative efficiency of the QW is $100 \%$, the value of $\eta_{r}$, which is the product of $\eta_{i}$ and $\eta_{\text {inj_below }}$, will be only $82 \%$. Since the value of $\eta_{\text {inj_below }}$, is structure dependent, QW lasers with poor active layer carrier confinement can be expected to have significantly lower values.

Note that the current injection efficiency values presented here are estimated values, as the actual current injection efficiency will depend on the actual recombination lifetime in the QW and barrier regions. In our analysis, the bimolecular recombination coefficients (B) in QW and barrier regions were assumed as $1.5 \times 10^{-10} \mathrm{~cm}^{3} / \mathrm{s}$, while the monomolecular recombination coefficients (A) for both QW and barriers were fixed at $10^{8} 1 / \mathrm{s}$. The Auger coefficients (C) are taken as $5 \times 10^{-30} \mathrm{~cm}^{6} / \mathrm{s}$ for both QW and barrier regions. By using these parameters, the recombination lifetime in the $\mathrm{QW}\left(\tau_{\mathrm{QW}}\right.$ total $)$ and barrier $\left(\tau_{b}\right)$ regions can be obtained.

The total carrier capture time from the separate confinement heterostructure $(\mathrm{SCH}) /$ barrier to $\mathrm{QW}\left(\tau_{\mathrm{bw}}\right)$ consists of primarily the carrier transport time $\left(\tau_{r}\right)$ following the treatment in [17], [19], which is calculated using the ambipolar carrier transport time [17], [19]. The quantum-capture time ( $\left.\tau_{\text {cap_QW }}\right)$ has been neglected in our analysis, as this term is much smaller in comparison to the $\tau_{\mathrm{r}}$ in the $\tau_{\mathrm{bw}}$ analysis [16], [17]. For our $0.98-\mu \mathrm{m}$-emitting structure, the $\tau_{\mathrm{bw}}$ is calculated as $9.85 \mathrm{ps}$. The thermionic escape lifetimes $\left(\tau_{e}\right)$ depend on the band offset, effective masses in the QW, thickness of the QW, and carrier density in the QW. In our analysis, the $\tau_{e}$ were computed following the treatment in [17], [20].
The conventional approach to determine the current injection efficiency is in the above threshold regime, using a cavity length analysis [6]. The above threshold current injection efficiency is typically quite high $(80 \%-95 \%)$, as determined from cavity length measurements. However, no experimental technique has yet been developed to extract the current injection efficiency in the below threshold regime. Thus, the simulated below threshold current injection efficiency is taken into account as a means to gauge the "true" internal efficiency of the QW material. If current injection efficiency below laser threshold is $\eta_{\text {inj_below }}$, then the spontaneous radiative efficiency per injected electron is greater than the ratio of internal radiative current to the total external injected current obtained directly from the ASE experiments by the factor of $1 / \eta_{\text {inj_below }}$. Thus, for the $0.98-\mu \mathrm{m}$ - and 1.2- $\mu \mathrm{m}$-emitting InGaAs QW lasers, we find the internal spontaneous radiative efficiency is in the range $47 \%-64 \%$ and $16 \%-24 \%$, respectively.

\section{CONCLUSION}

The peak modal gain versus current characteristics of $\mathrm{In}_{x} \mathrm{Ga}_{1-x} \mathrm{As}(\mathrm{x} \sim 0.2,0.4)$ QW lasers are extracted from 1) single pass gain spectra measurements on a segmented contact device and 2) conventional cavity length analysis. The values of $\mathrm{g}_{0}$ and $J_{\mathrm{tr}}$ extracted from gain spectra measurement are within $25 \%$ of the values extracted from cavity length analysis. The spectral emission properties of 0.98- and 1.2- $\mu \mathrm{m}$-emitting InGaAs QW media were compared using measurements of the ASE from multisegmented devices. A higher gain parameter, $g_{0}$, and lower transparency current density, $J_{\mathrm{tr}}$ is observed for the highly strained 1.2- $\mu$ m-emitting $\operatorname{In}_{0.4} \mathrm{Ga}_{0.6}$ As QW laser material The spontaneous radiative efficiency of the highly strained $\mathrm{In}_{0.4} \mathrm{Ga}_{0.6} \mathrm{As}$ QW structure is in the range of $16 \%$, suggesting that a reduction in threshold current density might be possible if material quality can be further improved and nonradiative recombination rate reduced. The role of the below threshold current injection efficiency is estimated from simulations to be in the range of $75 \%-85 \%$ at RT, suggesting that the internal radiative efficiency of the QW material is 20\% higher than the ratio of internal radiative current to external injected current extracted from ASE measurements.

\section{REFERENCES}

[1] M. Kanskar, T. Earles, T. J. Goodnough, E. Stiers, D. Botez, and L. J. Mawst, "73\% CW power conversion efficiency at $50 \mathrm{~W}$ from $970 \mathrm{~nm}$ diode laser bars," Electron. Lett., vol. 41, pp. 245-247, 2005.

[2] P. Crump, J. Wang, T. Crum, S. Das, M. Devito, W. Dong, J. Farmer, Y. Feng, M. Grimshaw, D. Wise, and S. Zhang, "High-power diode laser technology and applications III," Proc. SPIE, vol. 5711, no. 21, 2005.

[3] N. Tansu, J. Y. Yeh, and L. J. Mawst, "Extremely low threshold-current-density InGaAs quantum-well lasers with emission wavelength of 1215-1233 nm," Appl. Phys. Lett., vol. 82, pp. 4038-4040, 2003.

[4] D. J. Palmer, P. M. Smowton, P. Blood, J. Y. Yeh, L. J. Mawst, and N. Tansu, "Effect of nitrogen on gain and efficiency in InGaAsN quantumwell lasers," Appl. Phys. Lett., vol. 86, p. 071121, 2005.

[5] P. Blood, G. M. Lewis, P. M. Smowton, H. Summers, J. Thomson, and J. Lutti, "Characterization semiconductor laser gain media by the segmented contact method," IEEE J. Sel. Topics Quantum Electron., vol. 9 , no. 5, pp. 1275-1282, Sep./Oct. 2003.

[6] L. A. Coldren and S. W. Corzine, Diode Lasers and Photonic Integrated Circuits. New York: Wiley, 1995. 
[7] J. Minch, S. H. Park, T. Keating, and S. L. Chuang, "Theory and experiment of $\mathrm{In}_{1-x} \mathrm{Ga}_{x} \mathrm{As}_{y} \mathrm{P}_{1-y}$ and $\mathrm{In}_{1-x-y} \mathrm{Ga}_{x} \mathrm{Al}_{y}$ As long-wavelength strained quantum-well lasers," IEEE J. Quantum Electron, vol. 35, no. 5, pp. 771-782, May 1999.

[8] C. H. Henry, R. A. Logan, and F. R. Merritt, "Measurement of gain and absorption-spectra in algaas buried heterostructure lasers," J. Appl. Phys, vol. 51, pp. 3042-3050, 1980.

[9] C. H. Henry, "Theory of spontaneous emission noise in open resonators and its application to lasers and optical amplifiers," J. Lightw. Technol, vol. LT-4, no. 3, pp. 288-297, Mar. 1986.

[10] D. J. Palmer, P. M. Smowton, P. Blood, J. Yeh, L. J. Mawst, and N. Tansu, "2005 conference on lasers and electro-optics, CLEO," Opt. Soc. Amer., vol. 1, pp. 101-103, 2005.

[11] A. Al Muhanna, L. J. Mawst, D. Botez, D. Z. Garbuzov, R. U. Martinelli, and J. C. Connolly, "High-power (>10 W) continuous-wave operation from 100-/ $m u$ m-aperture 0.97-/ $m u$ m-emitting $\mathrm{Al}$-free diode lasers," Appl. Phys. Lett, vol. 73, pp. 1182-1184, 1998.

[12] P. W. A. Mcilroy, A. Kurobe, and Y. Uematsu, "Analysis and application of theoretical gain curves to the design of multiquantum-well lasers," IEEE J. Quantum Electron, vol. QE-21, no. 12, pp. 1958-1963, Dec. 1985.

[13] S. W. Corzine, R. H. Yan, and L. A. Coldren, "Theoretical gain in strained ingaas/algaas quantum-wells including valence-band mixing effects," Appl. Phys. Lett, vol. 57, pp. 2835-2837, 1990.

[14] E. Yablonovitch and E. O. Kane, "Reduction of lasing threshold current-density by the lowering of valence band effective mass," J. Lightw. Technol, vol. LT-4, no. 5, pp. 504-506, May 1986.

[15] P. M. Smowton and P. Blood, "The differential efficiency of quantumwell lasers," IEEE J. Sel. Topics Quantum Electron., vol. 3, no. 2, pp. 491-498, Apr. 1997.

[16] R. Nagarajan and J. Bowers, "Effects of carrier transport on injection efficiency and wavelength chirping in quantum-well lasers," IEEE $J$. Sel. Topics Quantum Electron., vol. 29, no. 6, pp. 1601-1608, Jun. 1993.

[17] N. Tansu and L. J. Mawst, "Current injection efficiency of InGaAsN quantum-well lasers," J. Appl. Phys, vol. 97, p. 054502, 2005.

[18] N. Tansu, Y. L. Chang, T. Takeuchi, D. P. Bour, S. W. Corzine, M. R. T. Tan, and L. J. Mawst, "Temperature analysis and characteristics of highly strained InGaAs-GaAsP-GaAs (lambda $>1.17 \mu \mathrm{m}$ ) quantum-well lasers," IEEE J. Quantum Electron., vol. 38, no. 6, pp. 640-651, Jun. 2002.

[19] G. W. Taylor and S. Jin, "Revisions to "transport solution for SCH QW laser diodes"," IEEE J. Quantum Electron., vol. 34, no. 10, pp. 1886-1889, Oct. 1998

[20] N. Tansu and L. J. Mawst, "The role of hole leakage in 1300-nm InGaAsN quantum-well lasers," Appl. Phys. Lett., vol. 82, pp. $1500-1502,2003$.

[21] S. Mogg, N. Chitica, R. Schatz, and M. Hammar, "Properties of highly strained InGaAs," Appl. Phys. Lett., vol. 81, pp. 2334-2336, 2002.

Gene Tsvid (S'07) was born in Moscow, Russia, in 1975. He received the M.S degree in physics from Moscow Institute of Physics and Technology, Moscow, Russia, in 1998.

Currently, he is a Research Assistant at the university of Wisconsin-Madison, where he is working toward the Ph.D. degree. His dissertation research involves development and characterization of novel semiconductor laser structures including interband quantum dot systems, type-I and type-II quantum-well systems, and unipolar intersubband quantum-cascade and quantum-box devices.

Jeremy Kirch (S'07) was born in Madison, WI, in 1983, and he received the B.S. degree in both electrical engineering and physics from the University of Wisconsin at Madison in May 2007. As a Roger Bacon Fellowship recipient, he is currently pursuing the M.S. degree in electrical engineering while developing MOCVD-grown metamorphic buffer layers on InP.

L. J. Mawst (M'88-SM'93) was born in Chicago, IL, in 1959. He received the B.S. degree in engineering physics and the M.S. and Ph.D. degrees in electrical engineering from the University of Illinois at Urbana-Champaign, in 1982, 1984, and 1987, respectively. His dissertation research involved the development of index-guided semiconductor lasers and laser arrays grown by MOCVD.
He joined TRW, Inc., Redondo Beach, CA, in 1987, where he was a Senior Scientist in the research center, engaged in design and development of semiconductor lasers using MOCVD crystal growth. He is co-inventor of the resonant optical waveguide (ROW) antiguided array and has contributed to its development as a practical source of high coherent power, for which he received the TRW Group Level Chairman's award. He developed a novel single- mode edge-emitting laser structure, the ARROW laser, as a source for coupling high powers into fibers. He is currently a Professor in the Electrical and Computer Engineering Department, University of Wisconsin-Madison, where he is involved in the development novel III-V compound semiconductor device structures, including vertical-cavity surface-emitting lasers (VCSELs), active photonic lattice structures, InGaAsN lasers, and high-power Al-free diode lasers. His current research on low-temperature MOCVD grown highly strained InGaAs and InGaAsN led to record low threshold current density diode lasers. He is a founder of Alfalight Inc, a Madison-based manufacturer of high-power diode lasers. He has authored or coauthored more than 175 technical papers and holds 19 patents.

Manoj Kanskar (M'00) received the Ph.D. degree in condensed matter physics from the University of Oregon, Eugene.

$\mathrm{He}$ is currently Vice President of Research and Development at Alfalight, Inc., Madison, WI, spearheading research and development of high-power, highefficiency, and high-brightness laser diodes and diode-pumped solid-state lasers. He has over 20 years of semiconductor, photonic and optoelectronics device design, fabrication and high-power laser system experience and has authored over 50 published papers and conference presentations.

Jason Cai received the Ph.D. degree in electrical engineering from University of Maryland, Baltimore County, in 2005.

After graduation, he joined Alfalight Inc., Madison, WI. His research interests include III-V compound semiconductor material epitaxy and processing, highpower wavelength stabilized lasers, high-power grating surface-emitting lasers, and optical communication systems.

Ronald A. Arif (S'08) received the B.S. degree in materials engineering from Nanyang Technological University, Singapore, in May 2002, and the M.S. degree in electrical engineering from the Department of Electrical and Computer Engineering, Lehigh University, Bethlehem, PA, in 2005, where he is currently working toward the $\mathrm{Ph} . \mathrm{D}$. degree in electrical engineering.

He was a Process Engineer with Agilent Technologies, Singapore, from 2002 to 2003. His research works include fundamental studies and novel approaches to improve radiative efficiency of visible gain media based on III-nitride semiconductor nanostructures for high-efficiency LEDs and lasers, in particular for solid-state lighting. He has also published more than 42 refereed journal and conference publications.

Nelson Tansu (M'08) was born in October 1977, and received the B.S. degree (with highest distinction) in applied mathematics, electrical engineering, and physics, and the Ph.D. degree in electrical engineering from the University of Wisconsin-Madison in 1998 and May 2003, respectively.

Since July 2003, he has been an Assistant Professor in the Department of Electrical and Computer Engineering (ECE) and the Center for Optical Technologies (COT), Lehigh University, Bethlemhem, PA. Since April 2007, he has been the Peter C. Rossin Assistant Professor of Electrical and Computer Engineering at Lehigh University. His research works cover the theoretical and experimental aspects of the physics of semiconductor optoelectronics materials and devices, the physics of low-dimensional semiconductor (nanostructure), and MOCVD epitaxy and device fabrications of III-nitride and III-V-nitride semiconductor optoelectronics devices on GaAs, InP, and GaN substrates. His teaching interests are in the areas of optoelectronics and photonics, semiconductor physics, applied quantum mechanics, and engineering electromagnetism. $\mathrm{He}$ has published widely in numerous refereed international journal and conference publications (more than 130), and he also currently holds several U.S. patents. He had served several times as a panel member for U.S. National Science Foundation, U.S. Department of Defense, and other agencies in thr U.S. and abroad. He has also given numerous lectures, seminars, and invited talks (more than 30) in universities, research institutions, and conferences in the U.S., Canada, Europe, and Asia. 
Dr. Tansu was a recipient of the Bohn Scholarship, the WARF Graduate University Fellowship, the Vilas Graduate University Fellowship, and the Graduate Dissertator Travel Funding Award, the 2003 Harold A. Peterson ECE Best Research Award (First Prize) at the University of Wisconsin-Madison.

Peter M. Smowton (SM'07) received the B.Sc. degree in physics and electronics and the Ph.D. degree in electrical engineering from the University of Wales, Cardiff, U.K., in 1987 and 1991, respectively, where he studied the frequency stabilization of laser diodes.

Currently, he is a Reader in Semiconductor Optoelectronics in the School of Physics and Astronomy at Cardiff University , Cardiff, U.K. His interests include the design, fabrication, and characterization of optoelectronic devices. Current research topics include quantum-dot lasers, recombination mechanisms in dilute nitride devices, high-power red emitters for photodynamic therapy, and the physics of InGaN light-emitting devices.

Dr. Smowton is a senior member of IEEE LEOS, and a member of the OSA and Institute of Physics (London, U.K.).
Peter Blood (F'01) received the Ph.D. degree from the University of Leeds, Leeds, U.K.

He subsequently worked at Philips Research Laboratories, Redhill, U.K. on various aspects of the electrical properties of semiconductors. During this period he spent some time as a visitor at Bell Laboratories, Murray Hill, NJ. Since 1983, he has been investigating the operation of quantum-well lasers and in 1990 he moved to the School of Physics and Astronomy, Cardiff University, Cardiff, U.K. The group has developed techniques for the observation of spontaneous emission from laser diodes as a means of probing their steady-state and dynamic behavior, and amplified spontaneous emission techniques for the measurement of absorption, gain, and spontaneous emission. Topics of current interest include gain and recombination processes in wide-gap nitrides and in self-assembled quantum-dot structures, and in particular phenomena associated with carrier localization in quantum-dot systems. He has given short courses and review tutorials on quantum confined lasers at many major international conferences, including CLEO and the International Semiconductor Laser Conference.

Dr. Blood is a Fellow of the Institute of Physics, a Fellow of IEEE/LEOS, and an Associate Editor for the IEEE JOURNAL OF QUANTUM ELECTRONICS. 